\title{
Experimental Investigation on the Kinematics of an Underactuated Mechanical Finger through Vision-Based Technology
}

\author{
C. COSENZA, R. BRANCATI, V. NIOLA*, S. SAVINO \\ Department of Industrial Engineering \\ University of Naples Federico II \\ Via Claudio 21 - 80125, Naples, Italy \\ ITALY
}

\begin{abstract}
Marker-less vision techniques represent a promising route in the development of experimental methods to study the kinematic and the dynamic parameters of mechanical systems. The knowledge of a great number of these parameters is a fundamental issue in the system behaviour analysis and represents an even more crucial aspect for underactuated mechanical systems. In this paper, a technique is proposed to identify the kinematics of the phalanges of an underactuated mechanical finger, starting from the acquisition of the finger point cloud data by means of contactless vision system devices. The analytical model identified allows to determine the underactuated finger configuration as function of the shaft rotation of the single motor of the mechanical system.
\end{abstract}

Key-Words: underactuated mechanical systems, computer vision devices, robotic hand, kinematics, geometric reconstruction algorithm

Received: April 21, 2021. Revised: January 1, 2022. Accepted: January 21, 2022. Published: February 9, 2022.

\section{Introduction}

In underactuated mechanisms, the main design criterion is the lower number of actuators in respect to the degrees of freedom. Underactuated mechanical hands are able to perform several grasping tasks due the shape adaptation ability to a wide range of objects [1]-[3]. At the same time, the complexity design reduction is associated to an easier control by the user and, furthermore, cost, weight and size decrease [4]. In previous papers, a model of a highly underactuated robotic hand, based on tendon driven fingers for prosthesis applications [5], [6], has been proposed. The robotic hand is composed of five fingers, each of which is made of three phalanges hinged to each other by pins, which represent the different articulations. A single actuator drives the movement of each finger by means of a differential system obtained with a selfadaptive pulleys mechanism. Moreover, the capabilities of this hand in grasping complex shape objects through a combined approach of theory, simulation and experiments have been already demonstrated and the first prototypes have been built [7], [8]. Underactuated mechanical systems require appropriate and suitable experimental methods to correctly monitor each component dynamics. Indeed, it is not a feasible solution to deal with multiple encoders assembled to each component; the encoders could modify the system behaviour due to the increase of weight. A possible route in this field is to adopt vision techniques to track the kinematics and the dynamics of these mechanical systems. Hand tracking has become of great interest due to several applications connected with hand gesture and sign recognition for humancomputer interaction, especially in virtual/augmented reality and game console control [9]. Hand pose estimation is a very challenging task since the hand is an articulated body with many degrees of freedom and it is not easy to deal in detail with its anatomical and physiological constraints [10]. For human hand detection, tracking and recognition, researchers have used visual markers, coloured gloves, and electromechanical/magnetic sensing devices, as well as other specifically designed hardware, such as wearable haptics [11], [12]. However, these sensors may hinder the natural hand and finger motions. Research groups in computer vision have also focused their attention to the development of markerless vision-based methods to detect, track and recognize hand poses, employing RGB-D depth sensors or Digital Image Correlation apparatus [13], [14]. Low-cost sensors, like the Microsoft $\odot$ Kinect or Intel $\odot$ RealSense, to measure the displacement of mechanical systems have been adopted even for control applications [15]-[26]. The output of a depth sensor is a point cloud, containing the three- 
dimensional coordinates of a point set, expressed within a certain coordinate system, representing the external surface of an object. Optimization algorithms or neural networks allow the hand detection and tracking due to three-dimensional or appearance models [27], [28]. A more complex scenario is associated with approaches proposed to deal with the detection and tracking of hand grasping or interacting with objects or with deformable object tracking [29]. As for the experimental analysis of underactuated components, researchers have already adopted vision methods with the limitation of qualitative analysis. To quantitatively assess the motion of mechanical fingers (i.e. joint angle rotations) researchers have also employed different methodologies: elastomeric sensors that can directly be deposited on the fingers of a prosthetic hand, flexible cables equipped with potentiometers, magnetic rotary encoders embedded between the proximal and medial phalanges [30][33]. In this paper, an experimental marker-less approach is proposed to quantitatively acquire the motion an underactuated mechanical finger. This approach exploits vision system acquisition instruments to measure the joint angles (i.e. the angles formed between the phalanges), at different actuator rotations. In this perspective, the main issue is to use a non-invasive methodology, able to not influence the behaviour of each low-mass phalanx $(5-10 \mathrm{~g})$ composing the finger. The use of sensors could affect the original motion of the robotic finger. A customized algorithm has been developed to build a geometrical model of the fully articulated robotic finger, starting from the output of a vision system device. Finally, the results of the methodology are used to write an analytical expression to describe each phalanx rotation as a function of the actuator rotation.

The study is organized as follows: in Section 2, the mechanical finger system has been presented; in Section 3, the experimental setup and the algorithm description; in Section 4, we have discussed the results for both the adopted instruments; in section 5 , the validation of the results in comparison to a non-vision method instrumentation and the analytical expression for phalanx rotations are shown; in Section 7, the main conclusions are pointed out.

\section{The Underactuated Mechanical Finger}

The mechanical finger, object of this study, is composed by three phalanges, the proximal, the medial and the distal, connected to each other and to the hand through three hinges. Inside the three phalanges, the actuator and the antagonist tendons slide allowing the finger closing and opening, respectively. Both the tendons are connected on one side to the distal phalanx and on the other side to the same actuator; so, it is possible to guarantee the correct closing sequence, which is necessary to facilitate grasping tasks. An elastic element, connected to the antagonist tendon, compensates for the inevitable different displacements of the two tendons, due to the different geometries of their paths, Figure 1.

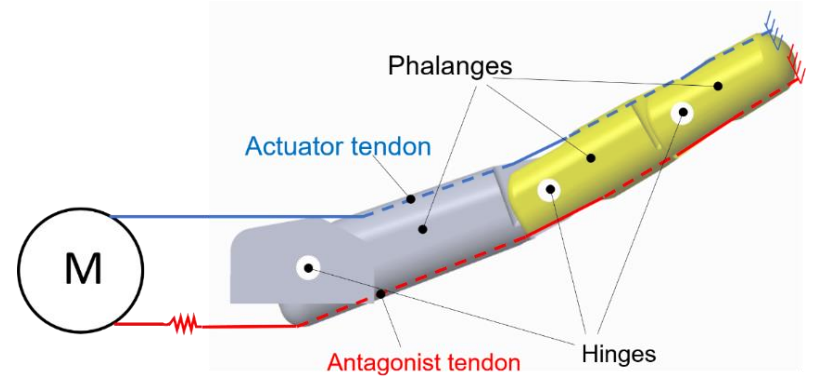

Fig. 1: Scheme of the underactuated finger.

By assembling several fingers, it is possible to realize an underactuated gripper. Activating all the fingers with a single actuator, it is possible to realize a strongly underactuated system. In this configuration, both the tendons of each finger are actuated by one actuator; two differential systems distribute the forces between the fingers in a fixed manner, but at the same time leave the fingers free to move to better wrap different shape objects.

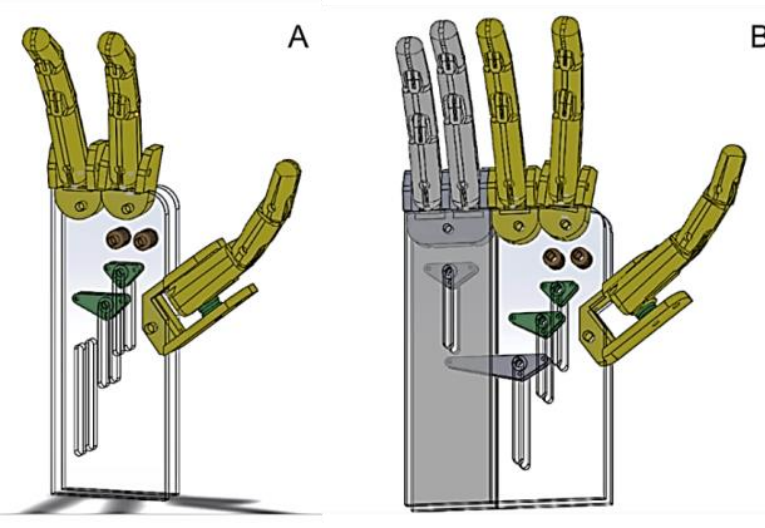

Fig. 2: CAD model of two underactuated grasping end-effectors: (a) the three-finger gripper; (b) the five-finger hand.

In Figure 2, it is possible to observe two different assemblies of the fingers to realize a three-finger gripper and a five-finger hand. The correct 
behaviour of each finger is crucial in the efficiency of the multi-finger gripper. For this reason, it is important to be able to analyse the real behaviour of the finger during the motion steps and avoid sensors that can alter the system dynamics. Thus, the approach that will be proposed in the following paragraphs aims to be non-invasive for the mechanical system.

\section{Description of the Proposed Approach}

The goal of the proposed approach is to acquire the finger phalanx rotations through vision devices and compute the relative angles between the phalanges. As a first step, it is necessary to acquire the $3 \mathrm{D}$ point data of the finger through vision devices. Afterward, the data must be purged of points not belonging to the finger itself and divided in three different point clouds representing the proximal, the medial and the distal phalanges. The point clouds of the phalanges are processed through an appropriately developed algorithm (hereafter the finger reconstruction algorithm), which aims to reconstruct the finger geometrical model by means of three cylinders. In this paper, we have acquired data with two different vision systems to test the reliability of the algorithm.

\subsection{Experimental Setup and Acquisition Instruments}

The experimental setup comprises of a mechanical finger, a controller unit and a servomotor. Figure 3 shows the whole test rig employed in this study. The finger is linked to a pulley by an inextensible wire that represents the tendon system. An analog servomotor sets the finger tendon displacement; the servomotor is controlled by a National Instruments myRIO Embedded Device controller. The experimental setup is also equipped with an incremental encoder, with 10000 pulses per revolution, to measure the motor angular position.

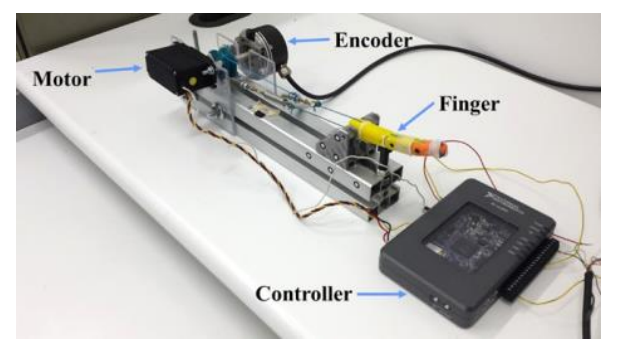

Fig. 3: Test rig of the single finger prototype.

Through the controller, it is possible to set a given motor shaft rotation. The motor shaft rotation produces the movement of the actuator and the antagonist tendons and consequently it determines a given finger configuration associated to the rotation of the three phalanges.

Two different instruments were employed to acquire the finger point clouds: a 3D laser scanner (ROMER Absolute Arm with integrated scanner, Hexagon Manufacturing Intelligence). The instrument resolution is $0.001 \mathrm{~mm}$ on the point cloud (Instrument 1); a white light scanner system (AICON MoveInspect XR8, Hexagon Manufacturing Intelligence) equipped with two high-resolution 8-megapixel digital cameras. The instrument resolution is $0.1 \mathrm{~mm}$ on the point cloud (Instrument 2).

The acquisitions performed with instrument 1 were made at a distance of a few centimetres from the surface of the finger. The data collected with the instrument 2 were obtained with acquisitions carried out at a greater distance from the finger between 30 and $40 \mathrm{~cm}$.

The finger point clouds were acquired in eleven static poses of the finger, in the range 0 to 180 degree of motor shaft rotation with 18-degree step. During the acquisition phase, the mechanical finger is under no load. The data overlaps acquired through both the instruments are shown in Figures 4 and 5.

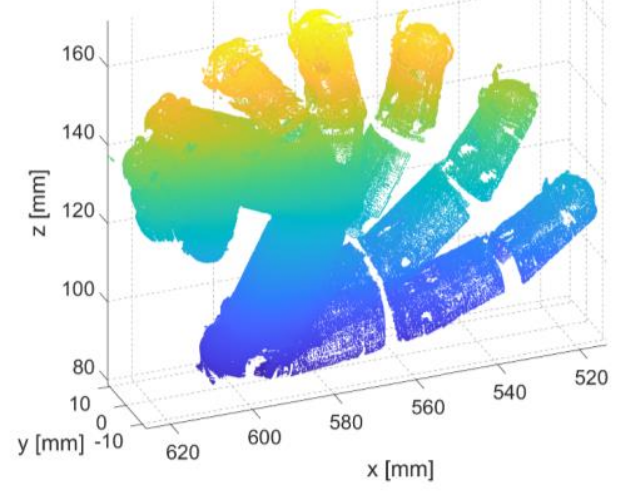

Fig. 4: Point cloud data overlap for Instrument 1.

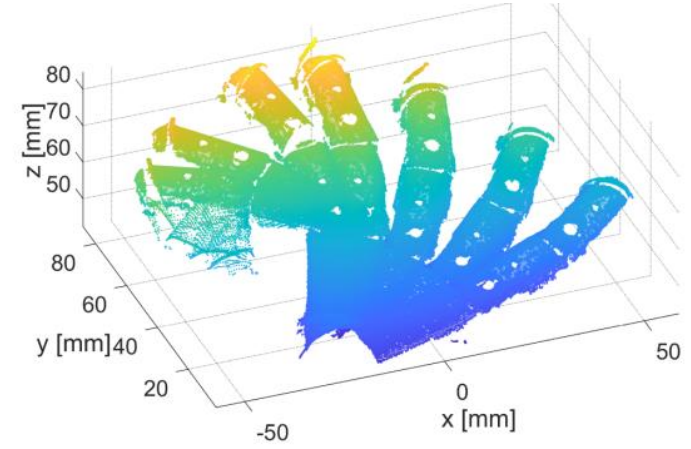

Fig. 5: Point cloud data overlap for Instrument 2.

In these two figures, the difference in the axis scale is due the different reference systems of the two instruments. 
The aim of this study is to estimate the finger phalanx rotations, using measurements obtained with non-contact equipment, independently of the instrument and the position of the instrument.

\subsection{Finger Reconstruction Algorithm: data processing}

The result of the acquisition is a point cloud of the finger external surface, in three-dimensional coordinates $(x, y, z)$, in the reference system of the instruments having the $\mathrm{z}$-axis in the observation direction.

As a preliminary process, it is necessary to remove from the point cloud those points that do not belong to the finger and to divide the point cloud in three subsets corresponding to the proximal, the medial and the distal phalanges. The 3D laser scanner, the instrument 1 , acquires the external surface of the three phalanges in three separate point clouds. Conversely, the instrument 2 acquires the external surface of the finger in an overall point-cloud. Since the acquisition gives also an image of the observed area, it is possible to obtain three different point clouds of the phalanges through image analysis with morphology operations based on color segmentation.

\subsection{Finger Reconstruction Algorithm: mathematical formulation}

The finger reconstruction algorithm aims to build the finger configuration as a simple 3D geometrical model composed of three cylindrical elements. The algorithm performs optimization procedures through the linear regression models and geometrical constraints.

At a given finger pose, the algorithm computes the following steps:

Starting from the point cloud data of each phalanx, consider the point $P_{x y z}^{i}=\left[\begin{array}{lll}x_{R}^{i} & y_{R}^{i} & z_{R}^{i}\end{array}\right]$ in the instrument reference system $O_{R} x_{R} y_{R} z_{R}$ and its orthogonal projection, $P_{x y}^{i}=\left[\begin{array}{ll}x_{R}^{i} & y_{R}^{i}\end{array}\right]$, in the plane $O_{R} x_{R} y_{R}$, that is normal to the instrument observation direction $z_{R}$. For each phalanx, compute the direction vector a that minimizes the maximum distance of the points $P_{x y}^{i}$ from the straight line, figure. 6 .

The direction $\mathbf{a}$ is determined through the parametric equation (1) representing the straight line passing through the centroid $P_{x y}^{0}=\left[\begin{array}{ll}x_{0} & y_{0}\end{array}\right]$ of the points $P_{x y}^{i}$.

$$
\left\{\begin{array}{c}
x\left(s_{1}\right)=x_{0}+s_{1} l_{1} \\
y\left(s_{1}\right)=y_{0}+s_{1} m_{1}
\end{array}\right.
$$

In the equation (1), $s_{1}$ is the parameter, while $l_{1}$ and $m_{1}$ are the vector a components. For each point $P_{x y}^{i}$, the equation (2) allows one to calculate the value of parameter $s_{1}^{i}$ corresponding to the intersection point between the straight line (1) and the perpendicular line to (1) passing through the point $P_{x y}^{i}$.

$$
s_{1}^{i}=\frac{l_{1}\left(x_{R}^{i}-x_{0}\right)+m_{1}\left(y_{R}^{i}-y_{0}\right)}{l_{1}^{2}+m_{1}^{2}}(2)
$$

Thus, the distance from the point $P_{x y}^{i}$ to the straight line (1) is obtained through the following expression:

$$
d_{1}^{i}=\sqrt{\left[x_{R}^{i}-x\left(s_{1}^{i}\right)\right]^{2}+\left[y_{R}^{i}-y\left(s_{1}^{i}\right)\right]^{2}}
$$

The maximum value of distances $\boldsymbol{d}_{\mathbf{1}}^{\boldsymbol{i}}$, as a function of $\boldsymbol{l}_{\mathbf{1}}$ and $\boldsymbol{m}_{\mathbf{1}}$, is minimized. The minimization outputs are the values $\boldsymbol{l}_{\mathbf{1}}$ and $\boldsymbol{m}_{\mathbf{1}}$ that allow to evaluate the unit vector

$$
\widehat{a_{x y}}=\left[\frac{l_{1}}{\sqrt{l_{1}^{2}+m_{1}^{2}}} \frac{m_{1}}{\sqrt{l_{1}^{2}+m_{1}^{2}}}\right]=\left[\begin{array}{ll}
a_{x} & a_{y}
\end{array}\right] \text { (4) }
$$

in the plane $\boldsymbol{O}_{R} \boldsymbol{x}_{R} \boldsymbol{y}_{\boldsymbol{R}}$.

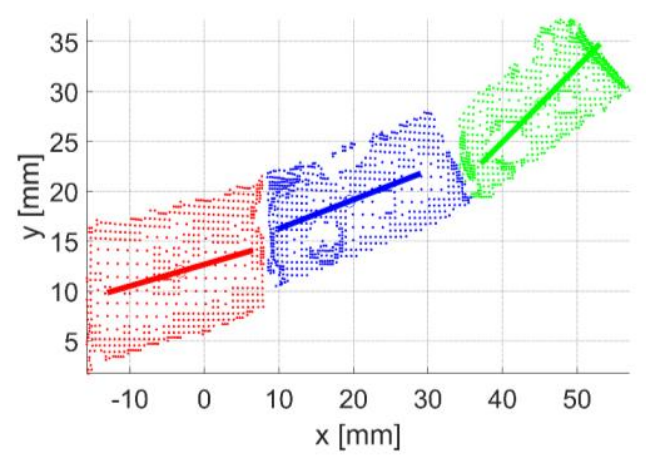

Fig. 6: Direction vectors for each phalanx in the xy-plane.

Consider the plane $O_{R} a z_{R}$, containing the directions $z_{R}$ and $\mathbf{a}$, orthogonal to the plane $O_{R} x_{R} y_{R}$, and project the points $P_{x y z}^{i}$ on this plane. The projected points are defined as $P_{a z}^{i}=\left[a^{i} z_{R}^{i}\right]$

Similarly, as in step 2 , it is possible to find the data direction vector $\mathbf{v}$ in the plane $O_{R} a z_{R}$. The direction $\mathbf{v}$ is computed considering the parametric equation (5) of the straight line passing through the centroid $P_{a z}^{0}=\left[a_{0} z_{0}\right]$ of the points $P_{a z}^{i}$.

$$
\left\{\begin{array}{l}
a\left(s_{2}\right)=a_{0}+s_{2} l_{2} \\
z\left(s_{2}\right)=z_{0}+s_{2} m_{2}
\end{array}\right.
$$


In (5) $s_{2}$ is the parameter, while $l_{2}$ and $m_{2}$ are the components of the vector $\mathbf{v}$.

Even in this case, for each point $P_{a z}^{i}$, by means of equation (6) it is possible to calculate the value of parameter $s_{2}^{i}$ corresponding to the intersection point between the straight line (5) and the perpendicular line to (5) passing through the point $P_{a z}^{i}$.

$$
s_{2}^{i}=\frac{l_{2}\left(a^{i}-a_{0}\right)+m_{2}\left(z_{R}^{i}-z_{0}\right)}{l_{2}^{2}+m_{2}^{2}}
$$

The distance from the point $P_{a z}^{i}$ to the straight line (5) is defined as:

$$
d_{2}^{i}=\sqrt{\left[a^{i}-a\left(s_{2}^{i}\right)\right]^{2}+\left[z_{R}^{i}-z\left(s_{2}^{i}\right)\right]^{2}}
$$

The maximum values of distances $d_{2}^{i}$, as function of $l_{2}$ and $\mathrm{m}_{2}$, are minimized and it is possible to calculate the unit vector

$$
\widehat{v_{a z}}=\left[\frac{l_{2}}{\sqrt{l_{2}^{2}+m_{2}^{2}}} \frac{m_{2}}{\sqrt{l_{2}^{2}+m_{2}^{2}}}\right]=\left[\begin{array}{ll}
v_{a} & v_{z}
\end{array}\right]
$$

in the plane $\boldsymbol{O}_{\boldsymbol{R}} \boldsymbol{a} \mathbf{z}_{\boldsymbol{R}}$. The direction $\mathrm{v}$ is the direction of the phalanx and its unit vector in the instrument reference system $O_{R} x_{R} y_{R} z_{R}$ is defined as:

$$
\widehat{v_{x y z}}=\left[\frac{a_{x}}{\sqrt{a_{x}^{2}+a_{y}^{2}+v_{z}^{2}}} \frac{a_{y}}{\sqrt{a_{x}^{2}+a_{y}^{2}+v_{z}^{2}}} \frac{v_{z}}{\sqrt{a_{x}^{2}+a_{y}^{2}+v_{z}^{2}}}\right]
$$

The unit vectors (9) associated to the three phalanges are used as the initial values to compute the cylinders.

The three cylinders, that approximate the phalanges, are calculated solving a non-linear curve fitting problem in least squares sense.

An optimization function describes the geometrical constraints of the finger configuration. The cylinders must assume the radii of the phalanges and the lengths corresponding to the distances between two consecutive hinges. Both the coplanarity errors of the cylinder axes and the fitting errors of each phalanx points from the respective cylinder surface are considered. A least squares minimization of such errors allows one to identify the unit vectors that represent the axes of the three cylinders. In this way, it is possible to reconstruct the finger configuration. The finger configuration reconstruction allows one to evaluate the relative rotations between the phalanges by calculating the relative rotations between the cylinder axes.

\section{Results of the Finger Reconstruction Algorithm}

The instrument 1 acquires the complete external surface of the finger. Fig. 7 shows the results of the phalanges reconstruction algorithm by means of an overlap between the point clouds acquired with instruments 1 and the surfaces of the reconstructed cylinders. Fig 7 shows three finger configurations, corresponding to three motor positions: 0,72 and 144 degrees.

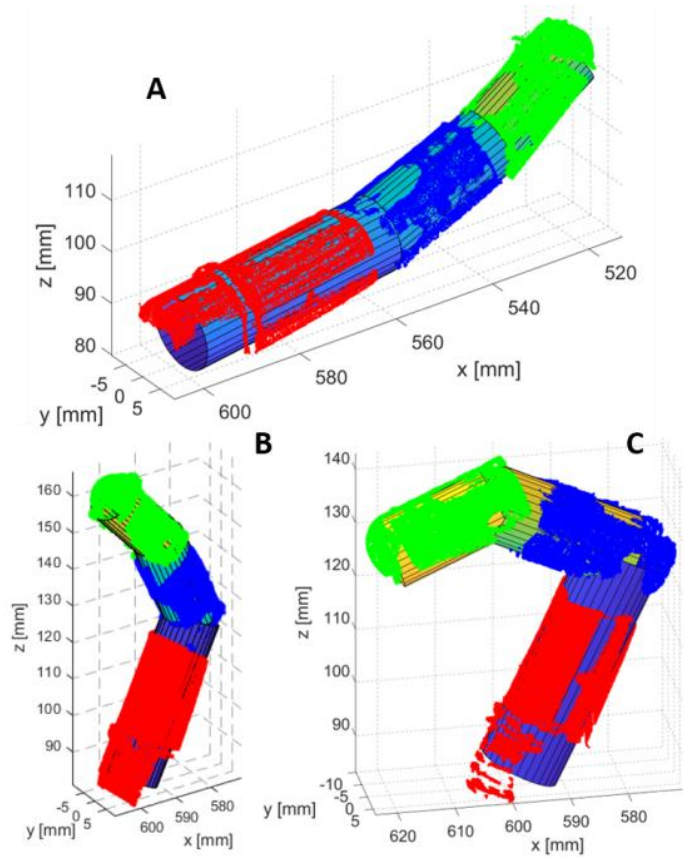

Fig. 7: 3D model reconstruction for each phalanx starting from the point cloud for three different motor shaft angle rotations: (A) 0 deg., (B) $72 \mathrm{deg}$. and (C) $144 \mathrm{deg}$. The point cloud is the output of the Instrument 1.

Figure 8 shows the output of the elaboration for data acquired with the instrument 2 for the same finger configurations. 


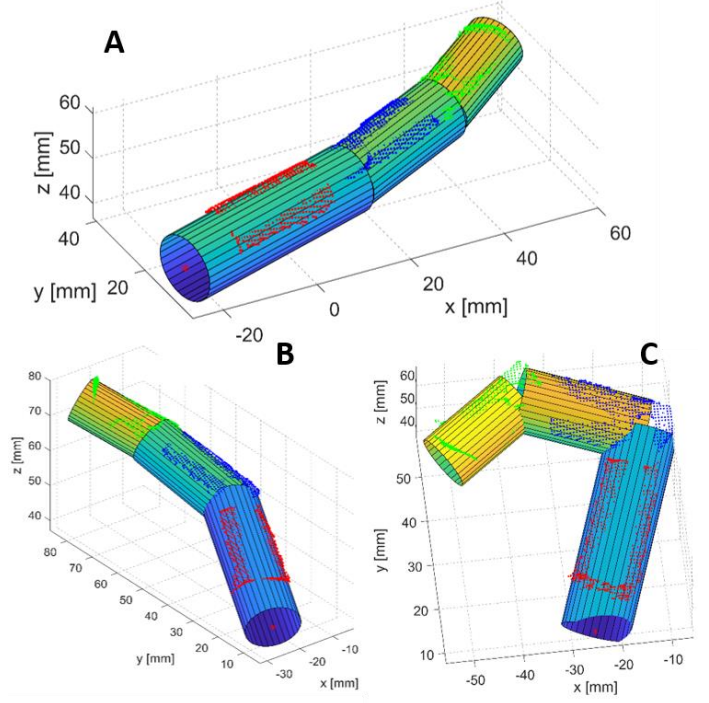

Fig. 8: 3D model reconstruction for each phalanx starting from the point cloud for three different motor shaft angle rotations: A) 0 deg., B) $72 \mathrm{deg}$. and C) $144 \mathrm{deg}$. The point cloud is the output of the Instrument 2.

It is possible to observe that the finger reconstruction algorithm is able to reproduce the finger configurations through the cylinders, even with the lower resolution data of instrument 2 , in which only a part of the phalanx surface has been acquired. For the eleven static poses, in the motor shaft rotation range [0-180] degrees, acquired through the two different vision instruments, the finger reconstruction algorithm allows to compute the rotations of the three phalanges.

\section{Validation of Results}

To verify the goodness of the obtained results, a comparison between the above results and those obtained with another no vision-based instrument, has been performed.

Thus, the angles of each phalanx have been also acquired by means of an inclinometer sensor (Dytran mod. 7546A1) equipped as showed in Figure 9.

Inclinometer measurements were performed in static finger poses and they were compared with the results obtained with the vision system data and the finger reconstruction algorithm.

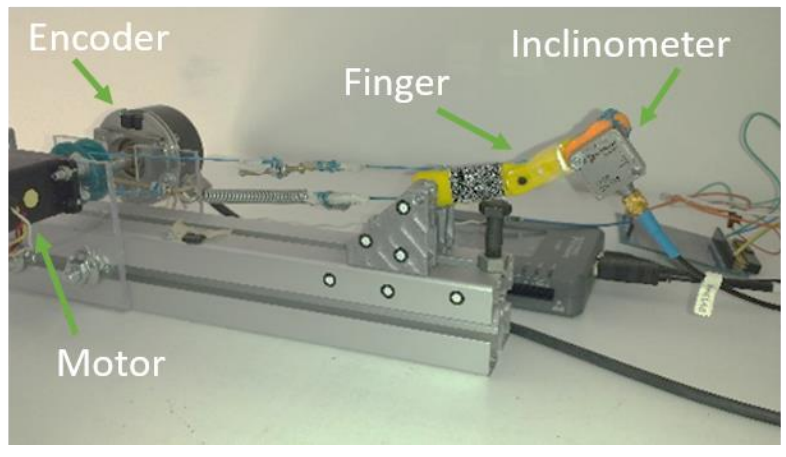

Fig. 9: Experimental setup for the inclinometer measurements for the distal phalanx.

The deployed inclinometer contains a variable capacitance MEMS device that allows measuring vibration data, including static inclination. The experimental test rig has been posed in plane and the inclinometer has been linked to each phalanx to acquire the absolute inclination, at the same motor shaft rotations actuated during the previous point cloud acquisitions. In this way, the rotation angles of the three phalanges, in the same static configurations above analyzed, have been measured. Figure 10 shows the measurements obtained from the inclinometer sensor in comparison with the rotations computed through the two vision instruments. The measured behavior of the phalanges is comparable for the three employed instruments.

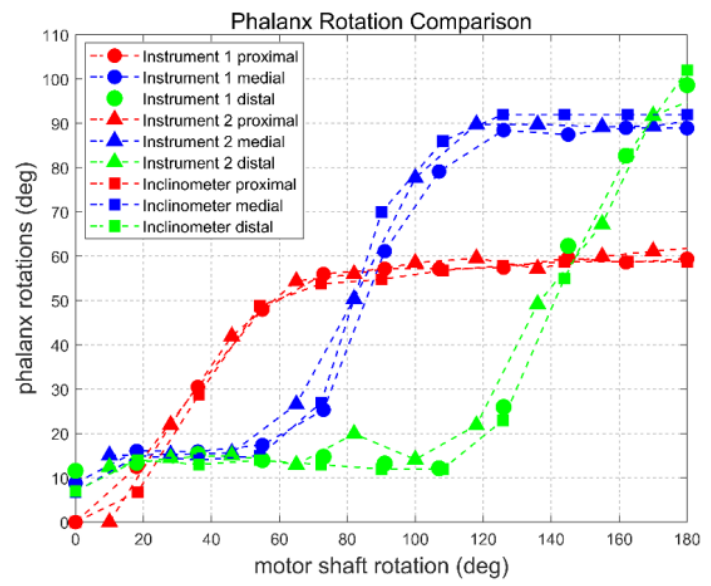

Fig. 10: Phalanx rotation angles as a function of the motor shaft rotation. Comparison between data acquired with the 3D laser scan (Instrument 1), the white light scanner system (Instrument 2) and the inclinometer sensor.

The standard deviation of the measurements obtained by the three instruments has been analysed. Figure 11 shows the standard deviations between 
the measurements for the three phalanges as a function of the motor shaft rotation.

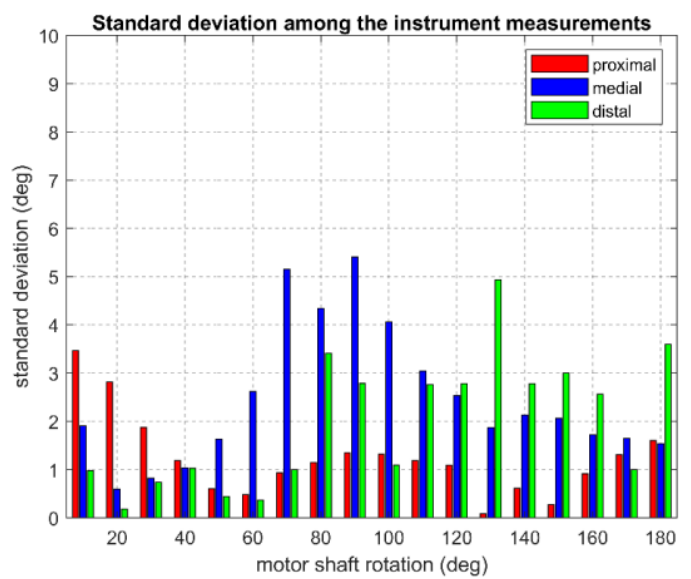

Fig. 11: Standard deviation of the measurements obtained by the three instruments.

The mean values of the standard deviations, in the motor shaft rotation range [0-180] degrees, are less than 3 degrees for all three phalanges, with a peak of about 5.5 degrees for the medial phalanx and a peak of about 5 degrees for distal phalanx. Since the medial phalanx is the central element of the underactuated finger system, it is affected by a greater error in the repeatability of the movements. This condition contributes to raising the standard deviation of the measurements of this phalanx.

The small values of the measurement deviations, performed with different instruments, indicate that the rotations evaluated by the finger reconstruction algorithm can be considered reliable, confirming the effectiveness of the proposed approach.

\section{Analytical Expression of Phalanx Rotations}

To verify the goodness of the obtained results, a comparison between the above results and those obtained with another no vision-based instrument, has been performed.

The values of phalanx rotations have been used to find an analytical expression which allows to compute the rotation of the three phalanges as a function of the motor shaft rotation and consequently of the actuator tendon displacement.

The experimental data reported in Figure 10 show that the trend of each phalanx as a function of motor shaft rotation could be described by a discontinuous function with three rectilinear sections and with six coefficients.
For the sake of simplicity, a sigmoid function, having a characteristic S-shaped curve or sigmoid curve, represents the best choice to describe the diagram of Figure 10.

A special case of the sigmoid curve is the logistic function whose general expression is:

$$
y(\theta)=\frac{\mathrm{a}}{1+\mathrm{b} \cdot e^{-c \cdot \theta}}+d(10)
$$

where $\mathrm{a}, \mathrm{b}, \mathrm{c}$ and $\mathrm{d}$ are the four function coefficients.

In this way, the data can be expressed through a continuous function and with a reduced number of coefficients.

Assume $\theta$ as the motor shaft rotation, it is possible to define three functions, $p(\theta), m(\theta), d(\theta)$, that are respectively the expressions of the behaviour of the proximal, medial and distal phalanges as function of $\theta$.

In the figures 12,13 and 14 , it is possible to observe the results of the fitting for the three functions. The expression (10) well describes the behaviour of all the three phalanges. The fitting coefficient of determination (R-squared) assumes values $0.9926,0.9945$ and 0.9863 respectively for the three phalanges proximal, medial and distal, as reported in Table 1.

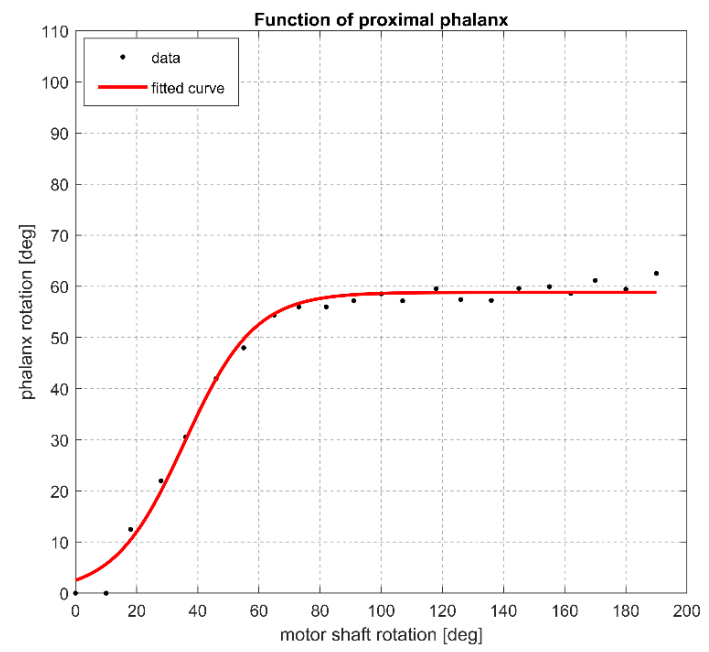

Fig. 12: Comparison between the experimental data and the fitting function $p(\theta)$ for the proximal phalanx. 


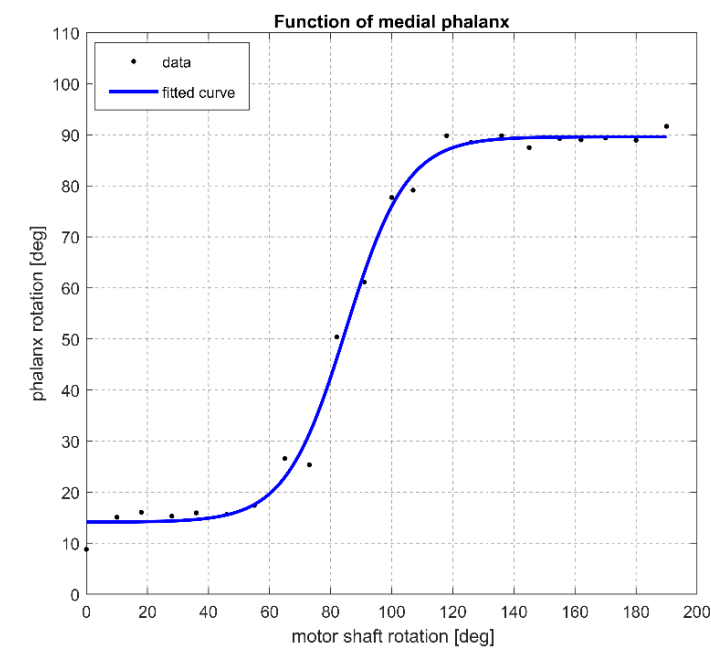

Fig. 13: Comparison between the experimental data and the fitting function $\mathrm{m}(\theta)$ for the medial phalanx.

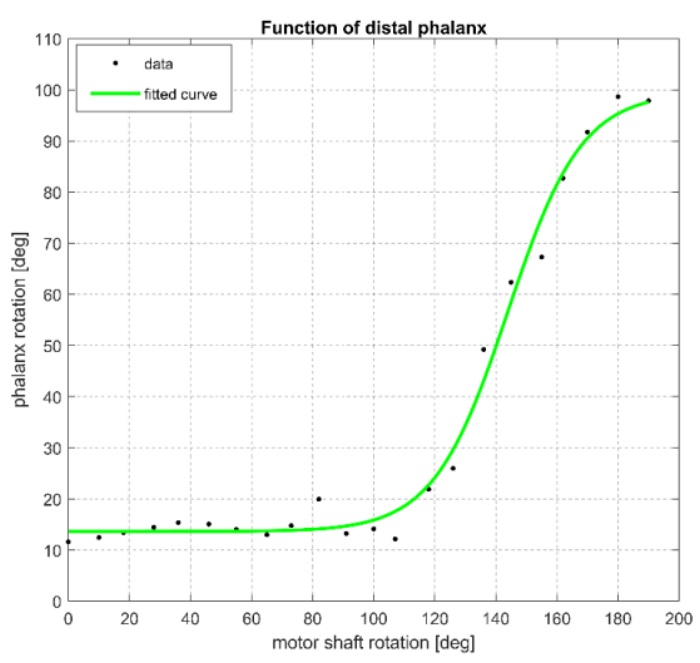

Fig. 14: Comparison between the experimental data and the fitting function $\mathrm{d}(\theta)$ for the distal phalanx.

Table 1 reports the coefficient values of the equation (8) for the functions associated to the three phalanx rotations.

The motor shaft rotation $\theta$ is related to the tendon displacement, therefore also the analytical functions $\mathrm{p}(\theta), \mathrm{m}(\theta)$ and $\mathrm{d}(\theta)$ can be related to the actuator tendon displacements.

It is worth to notice that the trend of these analytical functions, obtained through the proposed methodology, include most of the phenomena that characterize the real behaviour of the mechanical system.

The analysis of the rotation laws shows a different behaviour for the three phalanges. In figure 15 , the derivatives of the phalanx rotations with respect to the motor shaft rotation have been reported: they assume the highest value for the distal phalanx, while the lowest one for the proximal phalanx.

Table 1. Analytical expression coefficients of the phalanx rotations

\begin{tabular}{cccccc}
\hline & \multicolumn{5}{c}{ Logistic function coefficients } \\
\cline { 2 - 5 } $\begin{array}{c}\text { Phalanx } \\
\text { function }\end{array}$ & $\mathrm{a}$ & $\mathrm{b}$ & $\mathrm{c}$ & $\mathrm{d}$ & $\begin{array}{c}\mathrm{R}- \\
\text { squared }\end{array}$ \\
\hline $\mathrm{p}(\theta)$ & 63 & 15 & 0.08064 & -4.109 & 0.9926 \\
\hline $\mathrm{m}(\theta)$ & 75.48 & 5393 & 0.1011 & 14.12 & 0.9945 \\
\hline $\mathrm{d}(\theta)$ & 84.5 & $1.095 \mathrm{e}+06$ & 0.097 & 13.76 & 0.9863 \\
\hline
\end{tabular}

In the motor shaft rotation range $[0,180]$ degrees, five ranges can be identified; they correspond to finger configurations that are associated to different combinations of the three phalanx rotations, figure. 15 .

In particular:

1. $0-30$ degrees $\rightarrow$ proximal rotation

2. 30-90 degrees $\rightarrow$ proximal and medial rotations

3. 90-100 degrees $\rightarrow$ proximal, medial and distal rotations

4. 100-140 degrees $\rightarrow$ medial and distal rotations

5. 140-180 degrees $\rightarrow$ distal rotation

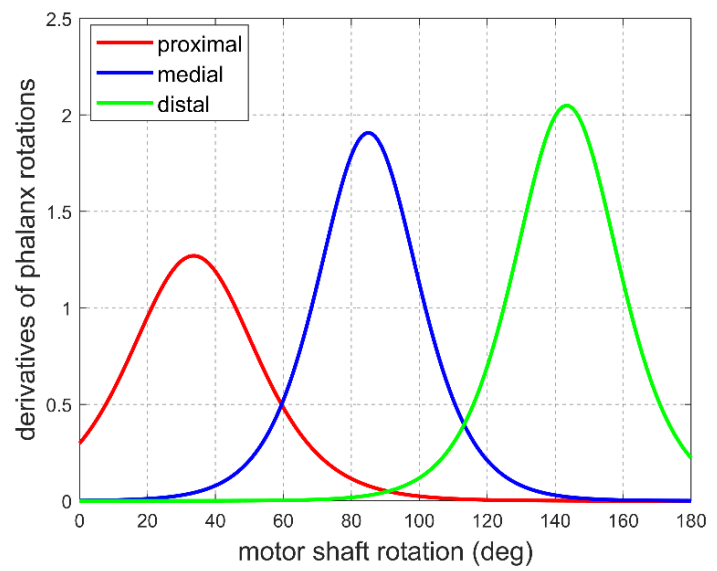

Fig. 15: Derivatives of the phalanx rotations with respect to the motor shaft rotation.

The analytical expressions $\mathrm{p}(\theta), \mathrm{m}(\theta)$ and $\mathrm{d}(\theta)$, represent the first step in defining a mathematical expression of the whole configuration of the underactuated mechanical finger and, therefore, of the underactuated mechanical hand as a function of the actuator tendon displacement. Such a 
mathematical expression can be a tool to improve the control of this mechanical system.

The kinematic finger model, with the reference systems placed in the hinges of each phalanx, [Xi, Yi, Zi], [Xp, Yp, Zp], [Xm, Ym, Zm], and in the finger-tip of the distal phalanx, [Xd, Yd, Zd], is showed in figure 16.

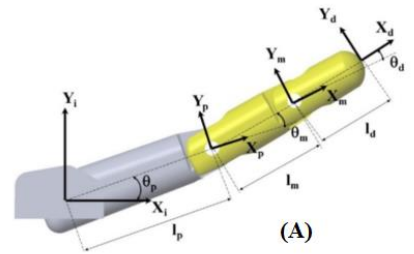

(A)

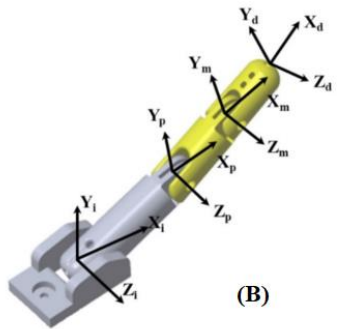

(B)
Fig. 16: Reference systems of kinematic finger model:(A) distal, (B) medial.

The coordinates of the ending point of the distal phalanx, $(\mathrm{x}, \mathrm{y}, \mathrm{z})$, coincide with the origin of the reference system $[\mathrm{Xd}, \mathrm{Yd}, \mathrm{Zd}]$, and in the reference system $[\mathrm{Xi}, \mathrm{Yi}, \mathrm{Zi}]$ are:

$\left\{\begin{array}{c}x=l_{p} \cdot \cos \left(\theta_{p}\right)+l_{m} \cdot \cos \left(\theta_{m}+\theta_{p}\right)+l_{d} \cdot \cos \left(\theta_{d}+\theta_{m}+\theta_{p}\right) \\ y=l_{p} \cdot \sin \left(\theta_{p}\right)+l_{m} \cdot \sin \left(\theta_{m}+\theta_{p}\right)+l_{d} \cdot \sin \left(\theta_{d}+\theta_{m}+\theta_{p}\right)(11) \\ z=0\end{array}\right.$

Where $1 \mathrm{p}, 1 \mathrm{~m}$, ld are proximal, medial and distal phalanx lengths (as above described, these lengths are the distances between two consecutive hinges); $\theta \mathrm{p}, \theta \mathrm{m}, \theta \mathrm{d}$ are the phalanx rotation angles.

This kinematic model allows to evaluate the fingertip position as function of the three phalanx rotations, but by means of the functions $p(\theta), m(\theta)$ and $\mathrm{d}(\theta)$, the fingertip position can be related to the only variable $\theta$, the motor shaft rotation, and therefore also to the actuator tendon displacement.

The kinematic model of the fingertip position in the reference system $[\mathrm{Xi}, \mathrm{Yi}, \mathrm{Zi}]$ is described by the equations (12) as a function of the motor shaft rotation $\theta$.

$x(\theta)=l_{p} \cdot \cos (p(\theta))+l_{m} \cdot \cos (p(\theta)+m(\theta))+l_{d} \cdot \cos (p(\theta)+m(\theta)+d(\theta))$ $\left\{y(\theta)=l_{p} \cdot \sin (p(\theta))+l_{m} \cdot \sin (p(\theta)+m(\theta))+l_{d} \cdot \sin (p(\theta)+m(\theta)+d(\theta))\right.$ $z(\theta)=0$

The fingertip displacements in the coordinate refence system [Xi, Yi, Zi] are showed in Fig. 17a), the fingertip trajectory, for a motor shaft rotation range of $[0,180]$ degrees, is showed in Fig 17b).

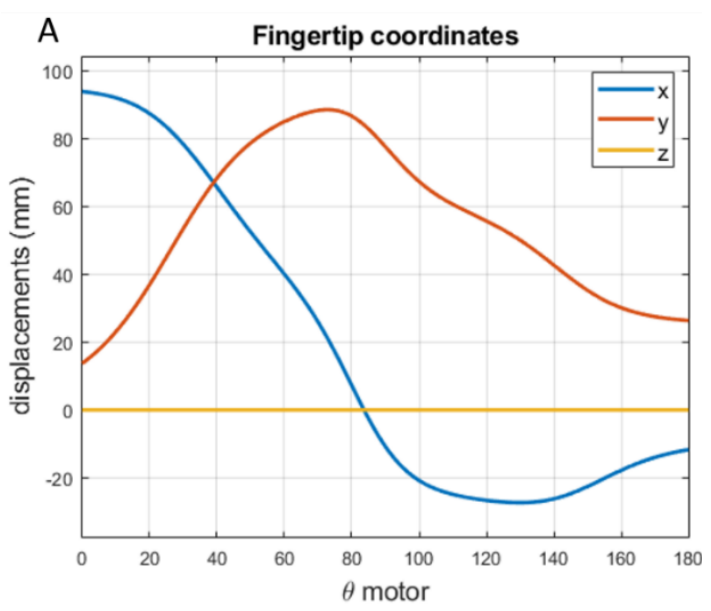

B

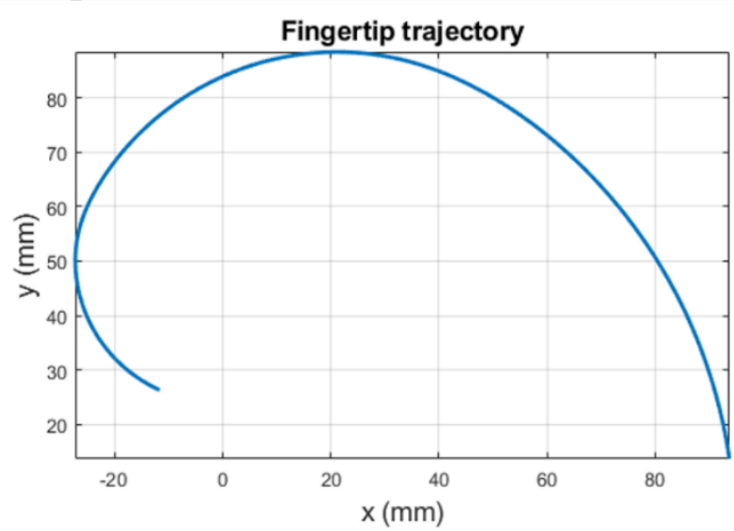

Fig. 17: (A) Coordinates of the fingertip in the reference system $[\mathrm{Xi}, \mathrm{Yi}, \mathrm{Zi}]$ as a function of the motor shaft rotation; (B) Fingertip trajectory for a motor shaft rotation range of $[0,180]$ degrees.

\section{Conclusions}

Experimental methods designed to monitor the kinematics and dynamics of underactuated systems are still lacking. Our study aims to propose an experimental technique to evaluate the configurations of an underactuated mechanical finger corresponding to different actuator rotations. An algorithm has been developed to reconstruct the three-dimensional shape of an underactuated mechanical finger, starting from the output of vision acquisition instruments. Two high-resolution instruments have been employed, namely a 3D laser scan and a white light scanner. For both the instruments, the algorithm reconstructs the threedimensional configuration of the finger in a very accurate manner.

The results of the finger reconstruction algorithm have also been validated by means of an inclinometer sensor that measures the inclinations of 
each phalanx. The standard deviations of the measurements performed with three different instruments confirm the effectiveness of the proposed methodology.

The main outcome of the activity is to obtain a kinematic model of an underactuated system like the finger of "Federica hand", starting from experimental data acquired without producing any interference on the behaviour of the system itself. The methodology results have been used to evaluate an analytical expression describing the behaviour of each phalanx as a function of the motor shaft rotation. Such analytical expression could be a powerful tool in the finger behaviour analysis in its possible tasks. It should be noted that this technique of acquisition of the finger configuration, is noninvasive and could be used even for the human fingers.

The methodology can be applied even to other kind of fingers, but it is necessary to acquire their point clouds during a closing sequence. For mechanical fingers with a different design, it is possible that the analytical functions describing the phalanx rotations could be different; but if only the geometrical parameters (phalanx radii and lengths) change, the above functions could be the same with different values of the coefficients. These aspects could be addressed in a subsequent study in which the procedure can be performed on different fingers in order to build an experimental database and try to obtain a generic mathematical expression.

This study represents a first step to develop a quantitative approach to study the kinematics and the dynamics of underactuated mechanical systems adopting marker-less vision techniques. Future developments will concern the extension of this methodology to the data acquired during the finger motion to measure its dynamics. Indeed, the analytical expression of the phalanx rotation obtained with the finger in static poses, is not able to describe the whole dynamic behaviour of the finger, i.e. the dissipative phenomena cannot be observed in static configuration.

\section{Acknowledgments:}

During this research, a valuable help was provided by Luca Sanseverino who was working for the bachelor's degree thesis, and the company HEXAGON Manufacturing Intelligence Italy. The authors thank Gennaro Stingo and Giuseppe Iovino (Department of Industrial Engineering), Mario Minocchi and Davide Marcone (IT Laboratory) for their technical support during the tuning stages of the test rig.

\section{References:}

[1] M. Tavakoli, B. Enes, J. Santos, L. Marques, and A. T. de Almeida, "Underactuated anthropomorphic hands: Actuation strategies for a better functionality," Rob. Auton. Syst., vol. 74, pp. 267-282, Dec. 2015.

[2] M. A. Saliba and C. W. De Silva, "Quasi-dynamic analysis, design optimization, and evaluation of a two-finger underactuated hand," Mechatronics, vol. 33, pp. 93-107, 2016.

[3] D. Esposito et al., "Evaluation of grip force and energy efficiency of the 'federica' hand," Machines, vol. 9, no. 2, 2021.

[4] D. Esposito, S. Savino, E. Andreozzi, C. Cosenza, V. Niola, and P. Bifulco, "The 'Federica' Hand," 2021.

[5] C. Cosenza, V. Niola, and S. Savino, "A mechanical hand for prosthetic applications: multibody model and contact simulation," Proc. Inst. Mech. Eng. Part $H$ J. Eng. Med., p. 95441191878754, 2018.

[6] V. Niola, C. Rossi, S. Savino, and S. Troncone, "An underactuated mechanical hand: A first prototype," in 23rd International Conference on Robotics in Alpe-Adria-Danube Region, IEEE RAAD 2014 - Conference Proceedings, 2015. doi: 10.1109/RAAD.2014.7002225

[7] C. Cosenza, V. Niola, and S. Savino, "Analytical study for the capability implementation of an underactuated three-finger hand," in Mechanisms and Machine Science, vol. 65, 2019, pp. 161-168.

[8] C. Cosenza, V. Niola, and S. Savino, Underactuated finger behavior correlation between vision system based experimental tests and multibody simulations, Mechanisms and Machine Science, vol. 66. 2019.

[9] S. S. Rautaray and A. Agrawal, "Vision based hand gesture recognition for human computer interaction: a survey," Artif. Intell. Rev., vol. 43, no. 1, pp. 1-54, 2012.

[10] A. Aristidou, "Hand tracking with physiological constraints," Visual Computer, pp. 1-16, 2016.

[11] V. Frati and D. Prattichizzo, "Using Kinect for hand tracking and rendering in wearable haptics," 2011 IEEE World Haptics Conf. WHC 2011, pp. 317-321, 2011.

[12] S. Melax, L. Keselman, and S. Orsten, "Dynamics based 3D skeletal hand tracking," I3D '13 Proc. ACM SIGGRAPH Symp. Interact. 3D Graph. Games, p. 184, 2013.

[13] S. Sridhar, F. Mueller, A. Oulasvirta, and C. Theobalt, "Fast and robust hand tracking using detection-guided optimization," in Proceedings of the IEEE Computer Society Conference on Computer Vision and Pattern Recognition, 2015, vol. 07-12-June, pp. 3213-3221.

[14] R. Brancati, C. Cosenza, V. Niola, and S. Savino, "Experimental measurement of underactuated robotic finger configurations via RGB-D sensor," in Mechanisms and Machine Science, vol. 67, 
2019, pp. 531-537.

[15] C. Cosenza, A. Nicolella, D. Esposito, V. Niola, and S. Savino, "Mechanical system control by rgbd device," Machines, vol. 9, no. 1, pp. 1-12, Jan. 2021.

[16] C. Cosenza, A. Nicolella, V. Niola, and S. Savino, "Experimental Approaches to Measure Displacements in Mechanical Systems Through Vision Devices," in Mechanisms and Machine Science, vol. 91, 2021, pp. 833-840.

[17] C. Cosenza, A. Nicolella, V. Niola, and S. Savino, "RGB-D Vision Device for Tracking a Moving Target," in Mechanisms and Machine Science, vol. 91, 2021, pp. 841-848.

[18] V. Niola, S. Savino, G. Quaremba, C. Cosenza, M. Spirto, A. Nicolella, "Study on the Dispersion of Lubricant Film From a Cylindrical Gearwheels with Helical Teeth by Vibrational Analysis," WSEAS Transactions on Applied and Theoretical Mechanics, vol. 16, pp. 274-282, 2021

[19] V. Niola and G. Quaremba, "The Gear Whine Noise and vibro-acoustic emission of gear-box," Proc. 11th WSEAS Int. Conf. Robot. Control Manuf. Technol. 11th WSEAS Int. Conf. Multimed. Syst. signal Process., pp. 138-143, 2011. ISBN: 978-960474276-9

[20] V. Niola, M. Spirto, S. Savino, and C. Cosenza, "Vibrational analysis to detect cavitation phenomena in a directional spool valve," Int. J. Mech. Control, vol. 22, no. 1, 2021.

[21] W. Huang, J. Lin, Z. Kong, and D. Ceglarek, "Stream-of-variation (SOVA) modeling - Part II: A generic 3D variation model for rigid body assembly in multistation assembly processes," $J$. Manuf. Sci. Eng. Trans. ASME, vol. 129, no. 4, pp. 832-842, 2007.

[22] C. Cosenza, V. Niola, and S. Savino, “A simplified model of a multi-jointed mechanical finger calibrated with experimental data by vision system," Proc. Inst. Mech. Eng. Part K J. Multibody Dyn., 2021.

[23] F. Pedersoli, S. Benini, N. Adami, and R. Leonardi, "XKin: An open source framework for hand pose and gesture recognition using kinect," Vis. Comput., vol. 30, no. 10, pp. 1107-1122, 2014.

[24] P. Coscia, F. A. N. Palmieri, F. Castaldo, and A. Cavallo, 3-d hand pose estimation from kinect?s point cloud using appearance matching, vol. 54. 2016.

[25] S. Pagano, R. Russo, and S. Savino, "A vision guided robotic system for flexible gluing process in the footwear industry," Robot. Comput. Integr. Manuf., vol. 65, p. 101965, Oct. 2020.

[26] C. Cosenza, V. Niola, and S. Savino, Modelling friction phenomena in an underactuated tendon driven finger by means of vision system device data, Mechanisms and Machine Science vol. 68. 2019.

[27] I. Oikonomidis, N. Kyriazis, and A. Argyros,
"Efficient model-based 3D tracking of hand articulations using Kinect," in Procedings of the British Machine Vision Conference 2011, 2011, p. 101.1-101.11.

[28] P. Krejov, A. Gilbert, and R. Bowden, "Guided optimisation through classification and regression for hand pose estimation," Comput. Vis. Image Underst., vol. 155, pp. 124-138, 2017.

[29] A. Petit, V. Lippiello, G. A. Fontanelli, and B. Siciliano, "Tracking elastic deformable objects with an RGB-D sensor for a pizza chef robot," Rob. Auton. Syst., vol. 88, pp. 187-201, 2017.

[30] Y. Park, I. Jo, J. Lee, and J. Bae, "A Dual-cable Hand Exoskeleton System for Virtual Reality," Mechatronics, vol. 49, pp. 177-186, 2018.

[31] R. Conti, E. Meli, and A. Ridolfi, "A novel kinematic architecture for portable hand exoskeletons," Mechatronics, vol. 35, pp. 192207, 2016.

[32] V. Sencadas, R. Mutlu, and G. Alici, "Large area and ultra-thin compliant strain sensors for prosthetic devices," Sensors Actuators, A Phys., vol. 266, pp. 56-64, 2017.

[33] S. H. Jeong, K. S. Kim, and S. Kim, "Development of a robotic finger with an active dual-mode twisting actuation and a miniature tendon tension sensor," in IEEE/ASME International Conference on Advanced Intelligent Mechatronics, AIM, 2016, vol. 2016-Septe, pp. 1-6.

\section{Contribution of Individual Authors to the Creation of a Scientific Article (Ghostwriting Policy)}

All the authors participated in the: conceptualization, investigation, writing, and revision phases. V.N. and C.C. took particular care of the experimental setup development and laboratory tests, R.B. developed the numerical models, and S.S. took care the results analysis.

\section{Sources of Funding for Research Presented in a Scientific Article or Scientific Article Itself}

"This research received no external funding".

\section{Creative Commons Attribution License 4.0 (Attribution 4.0 International, CC BY 4.0)} This article is published under the terms of the Creative Commons Attribution License 4.0 https://creativecommons.org/licenses/by/4.0/deed.en US 